\title{
The organic matrix of gallstones
}

\author{
D. JUNE SUTOR AND SUSAN E. WOOLEY \\ From the Department of Chemistry, University College, London
}

SUMMARY Dissolution of gallstones consisting of cholesterol, calcium carbonate, or calcium phosphate in different solvents left an amorphous organic gel-like substance (the matrix). Matrix from cholesterol stones could be colourless but was usually orange, yellow, or brown while that from calcium carbonate and calcium phosphate stones was almost invariably coloured black or dark brown. These pigments were also shown to be organic and amorphous. The amount of matrix present and its structure varied with the texture of the crystalline material. Irrespective of their composition, laminated pieces of material yielded compact laminated matrix of the same shape as the original piece and areas of loose crystalline material gave small pieces of non-cohesive matrix. Only large cholesterol crystals which usually radiate from the stone nucleus had no associated matrix.

In common with urinary stones and other pathological concretions investigated, gallstones consisting entirely or predominantly of cholesterol contain an organic matrix of mucopolysaccharide. King and Boyce (1959) dissolved out cholesterol and some pigment from pooled gallstones and found the overall composition of mucopolysaccharide present in the remaining material different from that in both urinary and salivary calculi. The intact gallstones were not analysed but dissolution studies suggested that the pool was $60 \%$ cholesterol. The presence of mucopolysaccharide in cholesterol gallstones was confirmed by Womack, Zeppa, and Irvin (1963) who stained polished sections of them.

Matrix has not been specifically sought in gallstones other than those of cholesterol nor has it been investigated for structural features. Studies on this material are necessary because it is an integral part of cholesterol gallstones at least. Its properties are also of importance since Danzinger, Hofmann, Schoenfield, and Thistle (1972) dissolved cholesterol stones in vivo by giving orally chenodeoxycholic acid, a compound unlikely to attack mucopolysaccharide. These could help to assess the likelihood of any residual matrix from 'stone dissolution' either being expelled by the gallbladder or remaining in that organ. In the latter case, such material could act as a nidus for further stone formation if the bile were in a supersaturated state. To help to solve these problems, we have undertaken a study of any matrix in gallstones of different composition and texture.

Received for publication 18 March 1974.

\section{Material and General Techniques}

At our disposal were several hundred gallstones whose crystalline composition had been thoroughly investigated by the $x$-ray powder method (Sutor and Wooley, 1971 and 1973). Each stone or piece of stone selected for study consisted entirely of one of the main constituents of gallstones, namely, cholesterol, calcium carbonate, calcium phosphate, calcium palmitate, or calcium bilirubinate. Pigment was, however, present in most samples.

In different experiments different solvents were used to remove the crystalline material. The presence or absence of matrix could be quickly established by dissolving cholesterol gallstones in organic solvents such as chloroform, and calcium carbonate and calcium phosphate stones in normal hydrochloric acid. These reactions were usually so quick or vigorous that the matrix often broke up and structural studies on this substance required much slower dissolution of the crystalline material. To this end cholesterol gallstones varying in weight from 11 to $26 \mathrm{mg}$ were each put in $12 \mathrm{ml}$ of a $4 \%$ aqueous solution of the sodium salt of chenodeoxycholic acid $(\mathrm{pH}=8 \cdot 1)$ and the solutions changed every six weeks, while calcium carbonate or calcium phosphate stones (5-41 mg in weight) were each placed in 12 $\mathrm{ml}$ of a $5 \%$ solution of the disodium salt of ethylenediaminetetra-acetic acid (EDTA) containing 0.4\% $\mathrm{w} / \mathrm{v}$ of formalin $(\mathrm{pH}=4.5)$ and the solutions changed every 10 days. Suitable solvents for calcium palmitate and calcium bilirubinate stones could not be found. Most of the calcium stones contained dark 
pigment some of which was soluble in water and some in a $5 \%$ solution of the tetrasodium salt of EDTA (pH adjusted to 7.9), but solvents could not be found which removed all the colouring matter, particularly the black pigment. These stones were alternated between the depigmenting and decalcifying solutions. The majority of experiments were carried out in a refrigerator $\left(1^{\circ} \mathrm{C}\right)$ but dissolution of cholesterol stones was also studied at room temperature $\left(23-27^{\circ} \mathrm{C}\right)$ and at $37^{\circ} \mathrm{C}$.

Some stones were weighed before and after dissolution in order to ascertain the amount of matrix present. All matrix was well washed in water and freeze dried before being weighed, analysed for carbon, hydrogen, nitrogen, and calcium, ashed or photographed by $x$-ray diffraction to determine whether it was amorphous or crystalline.

\section{Detailed Experiments and Results}

\section{CHOLESTEROL GALLSTONES}

Preliminary dissolution studies of cholesterol gallstones in chloroform showed that a gel-like substance (the matrix) was present in many stones and its structure varied with that of the crystalline material with which it was associated. The matrix could be colourless but it was usually orange, yellow, brown, or sometimes black. These observations were confirmed in the following experiments.

Five small gallstones from the same patient, each weighing 20-24 mg and having a thin surface band of fine-grained yellow material round a central area of almost colourless crystals radiating from the stone nucleus, were put in separate sodium chenodeoxycholate solutions in a refrigerator. Diffuseness of the outline of the stone, which was the first indication of the presence of matrix and resulted from the dissolution of the crystalline material leaving behind any associated gel, appeared within two weeks. The matrix as it was exposed flaked off and gradually broke into small yellow or colourless pieces. After about six months when the laminated surface bands and their associated matrix had gone, the stones took on a 'spiky' appearance. These spikes, which were crystals of cholesterol monohydrate, became more prominent as dissolution proceeded and finally broke up into a heap of single crystals which gradually dissolved. No matrix was present in this area. Dissolution of the stones took from seven to nine months.

To confirm the absence of matrix in areas consisting of radiating crystals of cholesterol, a few such pieces having linear dimensions of $1 \mathrm{~cm}$ were placed in chloroform and their dissolution was followed under the microscope. Matrix was only found in a small pigmented section of one corresponding to the original stone nucleus (magnification used $=150$ ).

Gallstones frequently consist of aggregates of crystals surrounded by laminated material. Small yellow-brown cholesterol stones (11-21 $\mathrm{mg}$ in weight) having these textures and from the same patient were placed in sodium chenodeoxycholate solution at $37^{\circ} \mathrm{C}$, room temperature, and $1{ }^{\circ} \mathrm{C}$. A cohesive area of almost colourless matrix was associated with the laminated bands (fig 1) and this sometimes separated from the stone in one piece but in all cases it gradually broke up (fig 2) eventually into small pieces. Dissolution of the central crystalline aggregates yielded only small incohesive pieces of brown matrix. The stones took two and a quarter to six months to dissolve. The solubility of cholesterol appeared to increase with temperature but because of variations in the weight of individual stones and the texture distribution in them, a quantitative estimation of this was not possible.

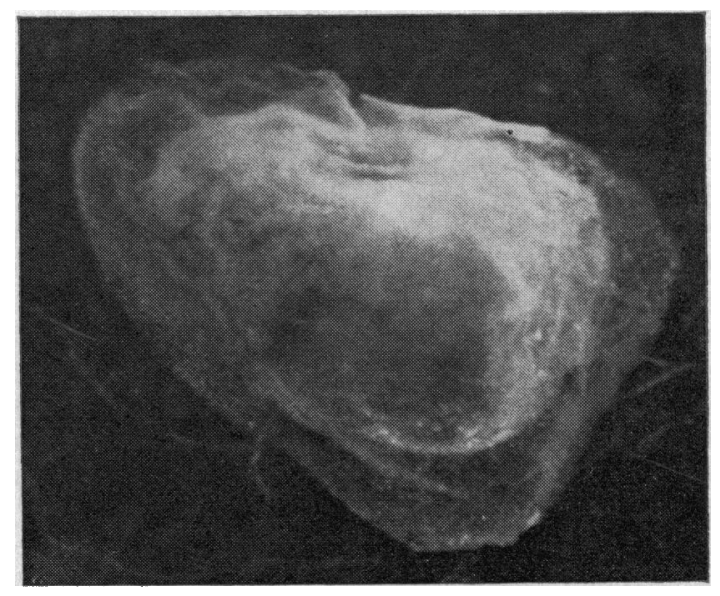

Fig 1 Cholesterol gallstone kept in sodium chenodeoxycholate for six weeks. Outer stone areas have dissolved leaving a colourless matrix but hard central core of cholesterol remains. Overall dimensions of material $6 \times 4 \mathrm{~mm}$. Approximate magnification in the figure $\times 10$.

A completely laminated brown cholesterol stone was cut into pieces weighing $18-26 \mathrm{mg}$ which were dissolved in sodium chenodeoxycholate at $37^{\circ} \mathrm{C}$, room temperature, and $1^{\circ} \mathrm{C}$. In all cases an exact replica in matrix of the original piece was obtained which was pigmented brown and tended not to disintegrate. The time for complete dissolution of the pieces was two-and-a-half to five months. The rate of dissolution appeared to increase with temperature 


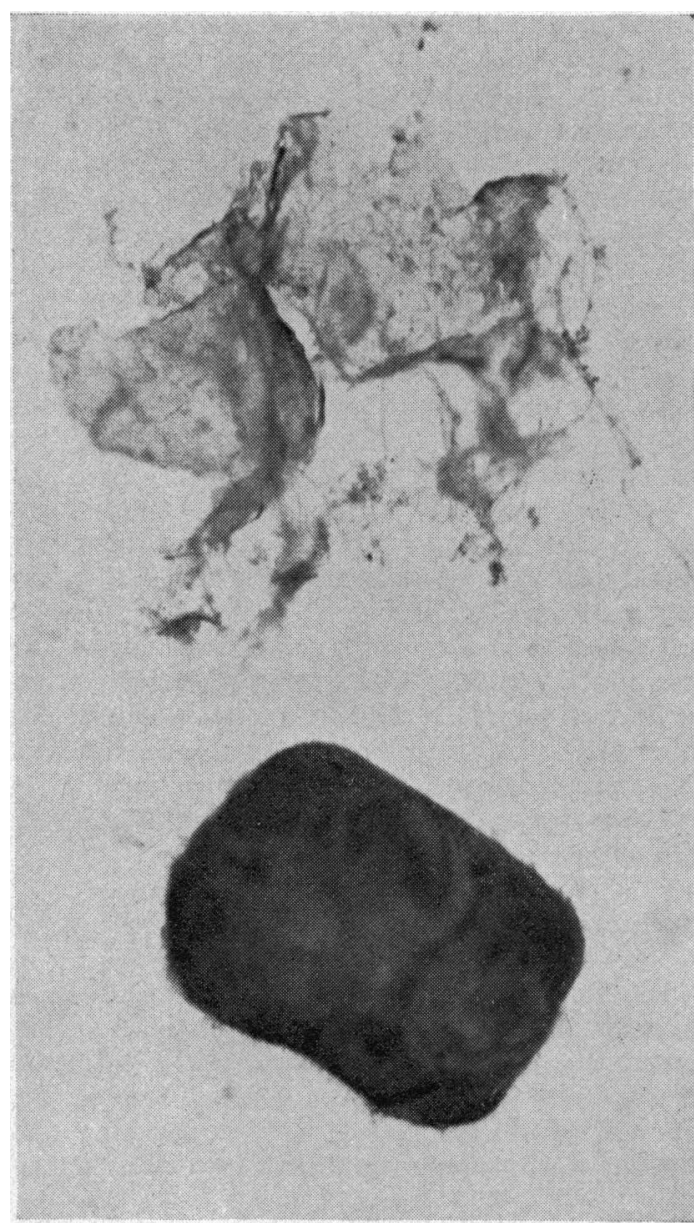

Fig 2 Shell of matrix (top) from cholesterol gallstone (bottom) kept in sodium chenodeoxycholate for 12 weeks. The matrix peeled off the stone and then broke up. Note diffuse areas of matrix on stone surface. Gallstone dimensions $4 \times 2.7 \mathrm{~mm}$. Approximate magnification in the figure $\times 10$.

but lack of uniformity in size and surface area of the specimens made comparisons difficult.

In the laminated pieces, the brown matrix accounted for $1 \%$ of their original weight and on heating gave a negligible amount of ash which was found to be calcium oxide by $x$-ray diffraction. Analysis figures for this material were $\mathrm{C}=58.01 \%, \mathrm{H}=$ $7.39 \%, \mathrm{~N}=11.41 \%$, but there was insufficient matrix from this and the other experiments to make further quantitative estimations. However, heating different samples of matrix gave only small amounts of ash suggesting that any metals were trace constituents. Several metals as well as calcium could be present but they were not unambiguously identified because not enough material was available to take the necessary type of $x$-ray photographs. Apatite (calcium phosphate) and calcium carbonate were sometimes found in these matrices which had not been in a decalcifying solution.

$X$-ray diffraction photographs showed that both the colourless and pigmented matrix were amorphous.

\section{GALLSTONES OF CALCIUM SALTS}

When calcium carbonate or calcium phosphate gallstones were dissolved in normal hydrochloric acid, a matrix was obtained which was almost invariably pigmented black and whose structure was related to that of the associated crystalline material. If, however, the gallstones contained much black pigment this tended to hold the matrix together and obscure structural features. These results were confirmed by the following slow decalcification experiments.

Six patients provided the calcium carbonate and four the calcium phosphate gallstones which were selected so that different textures and crystalline forms of the calcium salts were studied. The stones were black except for a group of brown calcium carbonate ones from one patient. All specimens were kept in the tetrasodium salt of EDTA for two months to remove a dark green pigment. They were then transferred to disodium EDTA and left to decalcify for nine and a half months. During this period no pigment was removed. When they were returned to tetrasodium EDTA, a further loss of green pigment occurred in many which was usually negligible after three months. The remaining material was then well washed in water for several weeks to remove the

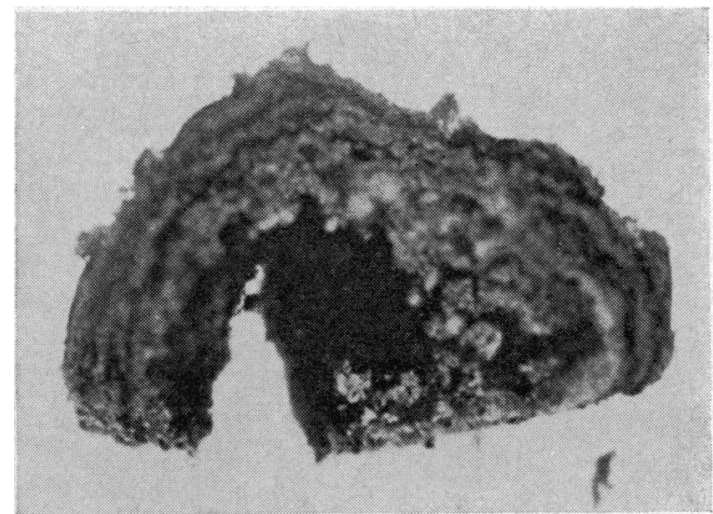

Fig 3 Cross section of laminated matrix from a laminated calcium carbonate gallstone. Dimensions $7 \times 4.7 \mathrm{~mm}$. Approximate magnification in the figure $\times 8 \cdot 8$. 


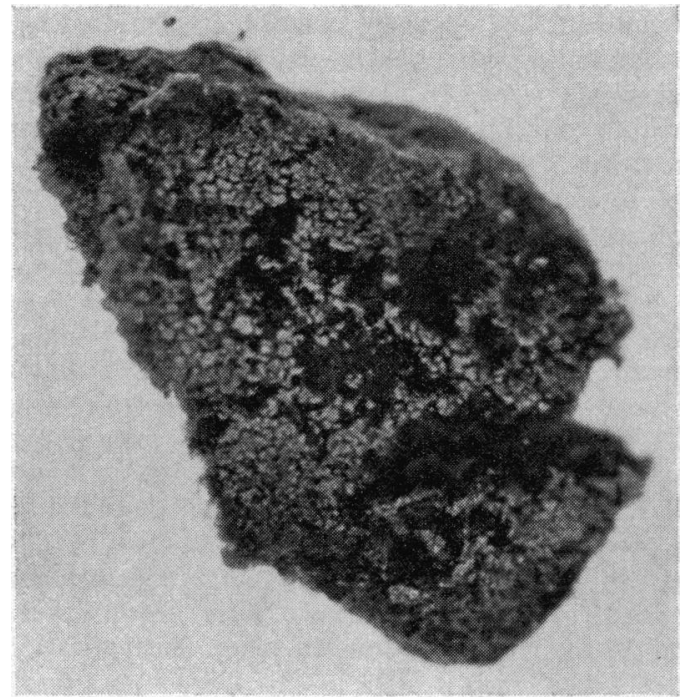

Fig 4 Surface detail of the matrix in figure 3. Dimensions $7 \times 4.8 \mathrm{~mm}$. Approximate magnification in the figure $\times 10 \cdot 5$.

EDTA solution and yellow and green pigments which were soluble in water.

The brown laminated calcium carbonate stones contained a yellow-brown laminated matrix (figs 3 and 4) which accounted for $9.3 \%$ of the original stone weight. Analysis figures for this material were $\mathrm{C}=56.81 \%, \mathrm{H}=6.04 \%, \mathrm{~N}=8.89 \%$.

The material obtained from the dissolution of the black calcium carbonate and calcium phosphate stones was usually gel-like and almost invariably pigmented black by an apparently insoluble compound (fig 5). Occasionally, firm areas remained in the matrix suggesting a localization of insoluble pigment or other material. In general, laminated stone areas gave laminated matrix which retained its cohesiveness and shape, while granular areas broke up in the decalcifying solution and yielded many small pieces of matrix. If, however, much black pigment was present in the stone, it obscured the underlying matrix structure (fig 5). The matrix and black pigment accounted for $12-27 \%$ of the original weight of gallstones from which a predominantly soft but pigmented matrix was obtained.

Carbon, hydrogen, and nitrogen analysis figures for 10 different specimens of pigmented matrix varied considerably: carbon $=47 \cdot 27-54 \cdot 69 \%$, hydrogen $=5 \cdot 06-6 \cdot 17 \%$, nitrogen $=6 \cdot 80-9 \cdot 52 \%$. Calcium was found in four out of six matrices analysed, but accounted for only $0 \cdot 3-2 \%$ of their weight. $X$-ray powder photographs of ashed matrices showed that other metals (not identified) could also be present in very small amounts.

These pigmented matrices gave amorphous $x$-ray diffraction patterns.

\section{Discussion}

A gel-like substance, the matrix, appears to be an essential constituent of all the gallstones studied irrespective of their composition. $X$-ray diffraction photographs showed that it was amorphous and analysis figures suggested it was organic, since calcium and other metals which were often present

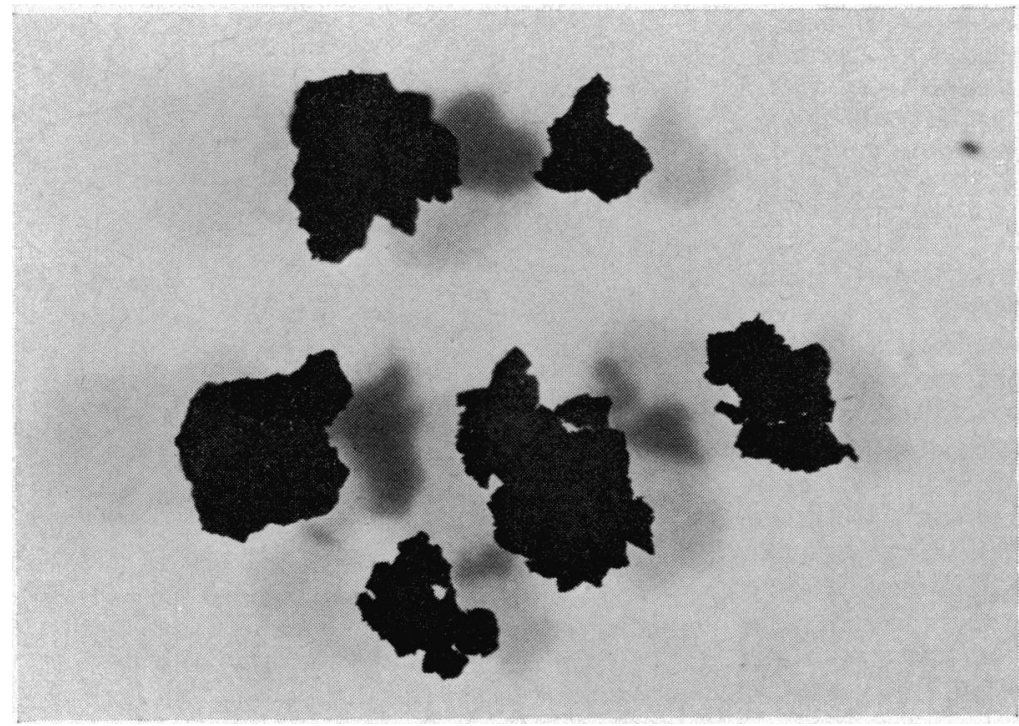

Fig 5 Black pigmented matrix from gallstones containing $40 \%$ pigment and $60 \%$ calcium phosphate. Approximate magnification in the figure $\times 5 \cdot 6$. 
occurred only in trace amounts. The relative proportions of carbon, hydrogen, and nitrogen could not be determined exactly because the matrix was usually pigmented when isolated under the conditions described here. Matrix from cholesterol stones was yellow, orange, or brown but very rarely black, while that from calcium carbonate and calcium phosphate varieties was black or dark brown. The colour of the matrix generally correlated with that of the stone but not with the presence or absence of trace elements. The pigments were not studied but analysis figures suggested that they too were organic compounds. This conflicts with Suzuki's view (1966) that the black insoluble pigment of gallstones is a metal complex of bilirubin but supports his earlier suggestion that it could be a high molecular weight polymer of bilirubin (Suzuki, 1965). This pigment is of particular interest since its occurrence would indicate a connexion between its formation and that of calcium phosphate and calcium carbonate.

Ashing matrix showed the presence of trace amounts of apatite and calcium carbonate in some from cholesterol gallstones which had not been in a decalcifying solution. Since neither of these compounds form during this process, they must be original stone constituents bound to the matrix otherwise they would have been removed as the cholesterol dissolved, but as trace constituents, they would not be detected when the stones were analysed by $x$-ray diffraction (Sutor and Wooley, 1971).

The amount and structure of the matrix present in the gallstones varied with the texture of the crystalline material. Matrix was associated with all crystalline textures except areas of large radiating crystals of cholesterol. Laminated stone areas gave a compact gel whose shape was an almost exact replica of the original material but loose crystalline aggregates yielded many small pieces of matrix. Estimations of the amount of matrix varied considerably, probably mainly because of contamination of this material by unknown amounts of pigments.

The close structural relationship between crystalline material and matrix in gallstones would indicate that their deposition is in some way connected, but the relative roles played by each in the nucleation and growth of a stone cannot usually be deduced. The exception is stone areas consisting of radiating cholesterol crystals in which the absence of observ- able matrix shows that such crystal growth occurred independently of this substance. On the other hand, Womack (1971) found that in Syrian hamsters fed a diet which resulted in cholesterol gallstone formation, mucus collected in viscous masses before the appearance of crystals and the earliest stones were soft and later ones more compact. It is likely then that crystal growth from supersaturated bile can occur in the presence or absence of a matrix.

Most cholesterol gallstones contain some matrix since very few consist entirely of the large radiating crystals. Although in most of our experiments this matrix tended to break up into small pieces as the crystalline material dissolved, it was not destroyed. After cholesterol gallstones have been dissolved in vivo by oral administration of chenodeoxycholic acid, should any of these pieces remain in the gallbladder, they can act as a nidus for crystal growth if the bile becomes supersaturated with respect to any compound. Furthermore, crystal growth on such a nidus will occur at a much lower level of supersaturation than is required for homogeneous nucleation and stone formation is therefore more likely to recur. A study of any matrix in gallstones formed after chenodeoxycholic acid therapy would be of great interest.

We thank the Medical Research Council for financial support, Mr J. Cresswell for taking the photographs and Weddel Pharmaceuticals Ltd for providing the chenodeoxycholic acid.

References

Danzinger, R. G., Hofmann, A. F., Schoenfield, L. J., and Thistle, J.L. (1972). Dissolution of cholesterol gallstones by chenodeoxycholic acid. New Engl. J. Med., 286, 1-8.

King, J. S., Jr., and Boyce, W. H. (1959). Analysis of renal calculous matrix compared with some other matrix materials and with uromucoid. Arch. Biochem., 82, 455-466.

Sutor, D. J., and Wooley, S. E. (1971). A statistical survey of the composition of gallstones in eight countries. Gut, 12, 55-64.

Sutor, D. J., and Wooley, S. E. (1973). The nature and incidence of gallstones containing calcium. Gut, 14, 215-220.

Suzuki, N. (1965). On black pigment of gallstones with special reference to comparison with bilirubin derivatives. Tohoku J. exp. Med., 85, 396-405.

Suzuki, N. (1966). On bilirubin-metal complex compounds in relation to black pigments of gallstones. Tohoku J. exp. Med., 90, 195-205.

Womack, N. A. (1971). The development of gallstones. Surg. Gynec. Obstet., 133, 937-945.

Womack, N. A., Zeppa, R., and Irvin, G. L. (1963). The anatomy of gallstones. Ann. Surg., 157, 670-686. 